\title{
Numerical and Experimental Study on Side-by-Side Darrieus Cascade Turbines Array
}

\author{
Ridho Hantoro ${ }^{1, a, *}$, I Ketut Aria Pria Utama ${ }^{2, b}$, Juniarko Pranandand ${ }^{3, c}$, Erna \\ Septyaningrum ${ }^{1, d}$ \\ ${ }^{1}$ Department of Engineering Physics, Institut Teknologi Sepuluh Nopember, Surabaya, Indonesia \\ ${ }^{2}$ Department of Naval Architecture, Institut Teknologi Sepuluh Nopember, Surabaya, Indonesia \\ ${ }^{3}$ Department of Marine Engineering, Institut Teknologi Sepuluh Nopember, Surabaya, Indonesia \\ a.hantoro@ep.its.ac.id,b.kutama@na.its.ac.id,c.juniarko@ne.its.ac.id, \\ d.erna.septya@gmail.com \\ *corresponding author
}

Keywords: array configuration, co-rotating, counter-rotating, Darrieus Straight Blade Cascaded, farm effectiveness

Abstract: The performance of array configuration is strongly influenced by the freestream velocity, turbines position, turbines spacing and turbines rotational direction. Many studies have been conducted to examine this issue. This current study aims to investigate the effect of turbines rotational direction on the array performance and determines the array configuration which provides farm effectiveness improvement. Vertical Axis Hydrokinetic Turbine-Darrieus Straight Blade Cascaded (VAHT-SBC) was employed in this study, as it has better performance and self-starting characteristic. Three configurations were tested and simulated, i.e. "co-rotating", "counter-rotating out" and "counter-rotating in". The array testing process and numerical study confirmed that the array performance was highly affected by the hydrodynamic interaction between turbines. The hydrodynamic interaction leads to the superposition of induced flow and freestream flow, producing the jet-type flow-field or canal effect. Hence, it enhances the array performance. Since "co-rotating" configuration has better farm effectiveness and independent to the incoming flow direction, this configuration is recommended for unstable source which has unpredictable incoming flow direction. The farm effectiveness of "co-rotating" configuration is 1.33 at freestream velocity of $1.3 \mathrm{~m} / \mathrm{s}$ and cross-stream distance of 1.5D.

\section{Introduction}

The developing class of renewable energy, hydrokinetic energy is a promising technology for hydro energy harvesting industry since it is high reliability and predictability. Hydrokinetic technology has been developed by many researches. There are two methods for hydro energy conversion, i.e. hydrostatic and hydrokinetic. The former (hydrostatic) utilizes head of water (potential energy) to generate electricity. The latter (hydrokinetic) extracts the kinetic energy with zero head, generally using hydrokinetic turbine [1]. 
Due to the high energy demand, the hydrokinetic turbines are installed in the form of turbine array configuration. Turbine array configuration is the arrangement of several turbines in rows and columns, aiming to optimize the energy harvesting process. The performance of turbine array is represented by the value of farm effectivity, which highly depends on the performance of each turbine. In many references, farm effectiveness is known as farm efficiency [2]. Due the complexity of hydrodynamic interaction among turbines, determination of the appropriate configuration seems to be difficult. The farm effectiveness is affected by incoming flow velocity, turbine position and turbine rotational direction [3], [4]. It also depends on the characteristic of canal or installation fields [3]. Ye Li declared that the farm effectiveness is the ratio of the power generated by array $(\varepsilon)$ and the power produced by $\mathrm{N}$ stand-alone turbine $\left(P_{\text {refffarm }}\right)$, as expressed in equation (1) - (3). $P_{\text {ref.farm }}$ does not only represent the power output of $\mathrm{N}$ stand-alone turbine but also the power output where no hydrodynamic interaction exists between turbine.

$$
\begin{gathered}
\varepsilon=\frac{P_{\text {farm }}}{P_{\text {ref.farm }}} \\
P_{\text {ref.farm }=N . P_{s}} \\
P_{\text {farm }}=\sum_{i}^{N} P_{i}
\end{gathered}
$$

As mentioned above, the performance of hydrokinetic array is highly affected by the flow characteristic around turbines. The major consideration for determining turbines position in array is the characteristic of near wake, especially near wake of upstream turbine [5]. Hence, the hydrokinetic turbine array should be designed in the appropriate configuration by considering the characteristic of flow around the turbine and the near wake. Many researches have been conducted to observe the flow around turbine, either for Horizontal Axis Hydrokinetic Turbine (HAT) or Vertical Axis Hydrokinetic Turbine (VAT). Research which is conducted by Conzemius confirmed that the rotational direction of vortex in the wake region has opposite direction to the turbine rotational direction [6]. Based on this research, Wei Yuan tested the performance of array which consists of many turbines with different rotational direction. The testing result showed that $\mathrm{CCW}$ downstream turbine which is operated in the wake region of $\mathrm{CW}$ upstream turbine generates greater power than $\mathrm{CW}$ downstream turbine which is operated in the wake of $\mathrm{CW}$ upstream turbine [7]. Adding counter-rotating turbines in the downstream region is an alternative solution for enhancing energy production [8]. Moreover, the cross-stream velocity ( $\mathrm{Vy}$ ) of a vertical axis turbine has positive direction on the windward and negative on the leeward [9]. These phenomena should be considered for array turbine design. The array turbine installation should be adjusted to the flow characteristic around turbine, in which the extraction process performs optimally. This study focused on the effect of rotational direction on the array performance. Furthermore, it also determined the array configuration which enhances the farm effectiveness

\section{Turbine and Array Design}

Due to its ability to extract energy from low velocity resource, Vertical Axis Hydrokinetic Turbine (VAHT) is known as promising technology for hydro energy harvesting. VAHT is an omnidirectional device since it could extract energy from various direction. It prefers for installation in the location where the incoming flow could not be predicted or change with season. Hence, this technology is recommended for ocean current energy harvesting system. This technology does not require yaw 
mechanism and easy to maintenance, leading to low maintenance cost [9]-[12]. But, the improvement for this technology is required since it has low efficiency and poor self-starting.

The lack of efficiency and self-starting characteristic motivate many researchers to keep on developing vertical axis turbine. Many vertical axis turbine that has been proposed have Cp around $0.19-0.47$ [13]-[15]. The most widely used vertical axis turbine, Darrieus straight blade has higher efficiency among others. Moreover, it is easy to fabricate.

The previous study proposed the novel design of Darrieus turbine which is called Vertical Axis Hydrokinetic Turbine - Darrieus Straight Blade Cascaded (VAHT-SBC), as depicted in Figure 1. This turbine is the development of conventional Darrieus Turbine. The utilization of cascaded-blade could gain more torque without enlarging the turbine dimension. VAHT-SBC consists of three arms, with 3 blades in each arm. It has passive pitch-mechanism in its outer blade, aiming to improve the selfstating ability. This mechanism provides freedom of blade to adjust the pitch angel in the range of $20^{\circ} \leq \beta \leq 20^{\circ}$, where $\beta$ is pitch angle.

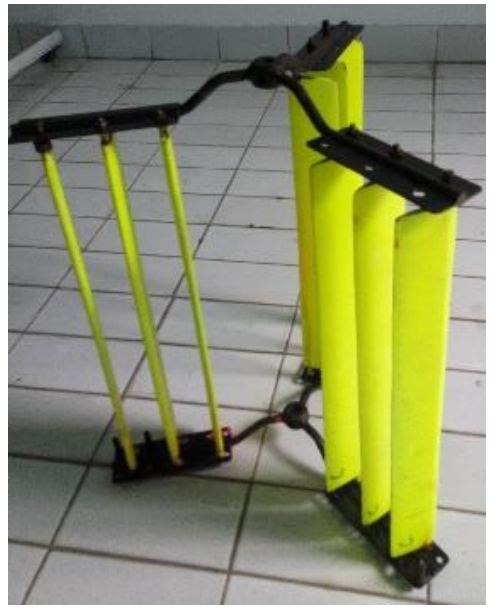

Figure 1. Vertical Axis Hydrokinetic Turbine-Darrieus Cascaded (VAHT-SBC)

NACA 0018 was selected as its hydrofoil, as this type of hydrofoil provided the best performance for vertical axis turbine application. The cord length and aspect ratio are $0.05 \mathrm{~m}$ and 8 , respectively. The aspect ratio is the ratio of span (turbine's high) to the cord length. H/D is 1 , which describe the ratio of turbine high and diameter.

To achieve production targets, modern hydrokinetic power plants utilize several turbines to harvest hydro energy. The turbines are arranged in a configuration, called turbines array. This study proposed 3 different configurations of side-by-side array turbine, i.e. "co-rotating configuration", "counter-rotating out configuration" and "counter-rotating in configuration". "Co-rotating configuration" is a configuration which consists of two clockwise turbines. Meanwhile, "counterrotating out configuration" is Turbine A on the left side rotates counter-clockwise (CCW) and Turbine B on the right side rotates clockwise $(\mathrm{CW})$. And "counter-rotating in" is Turbine A placed on the left side rotates clockwise $(\mathrm{CW})$ and Turbine B on the right side rotates counter-clockwise (CCW). Schematics of each configuration is illustrated in Figure 2. 


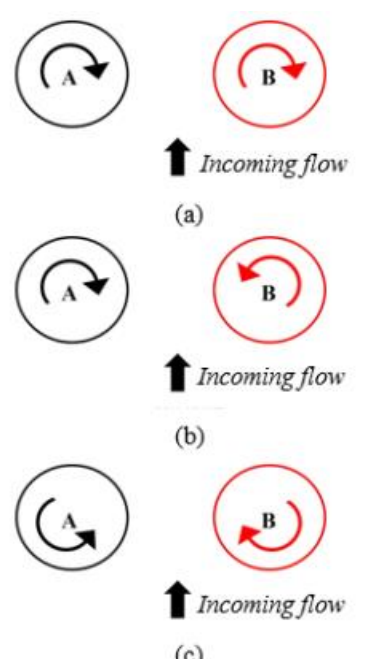

(c)

Figure 2. Schematic of turbine arrays: a. co-rotating configuration, b. counter-rotating out configuration, c. "counter-rotating in" configuration

\section{Research Methodology}

This study used two complementary methods, i.e. experimental (turbine testing process) and numerical simulation process. The numerical simulation was conducted to gain deeper information which could not be obtained from experimental process. At the beginning, the array turbines configuration was designed, followed by the fabrication process. The turbine design used in this study was turbines which is developed by the previous study.

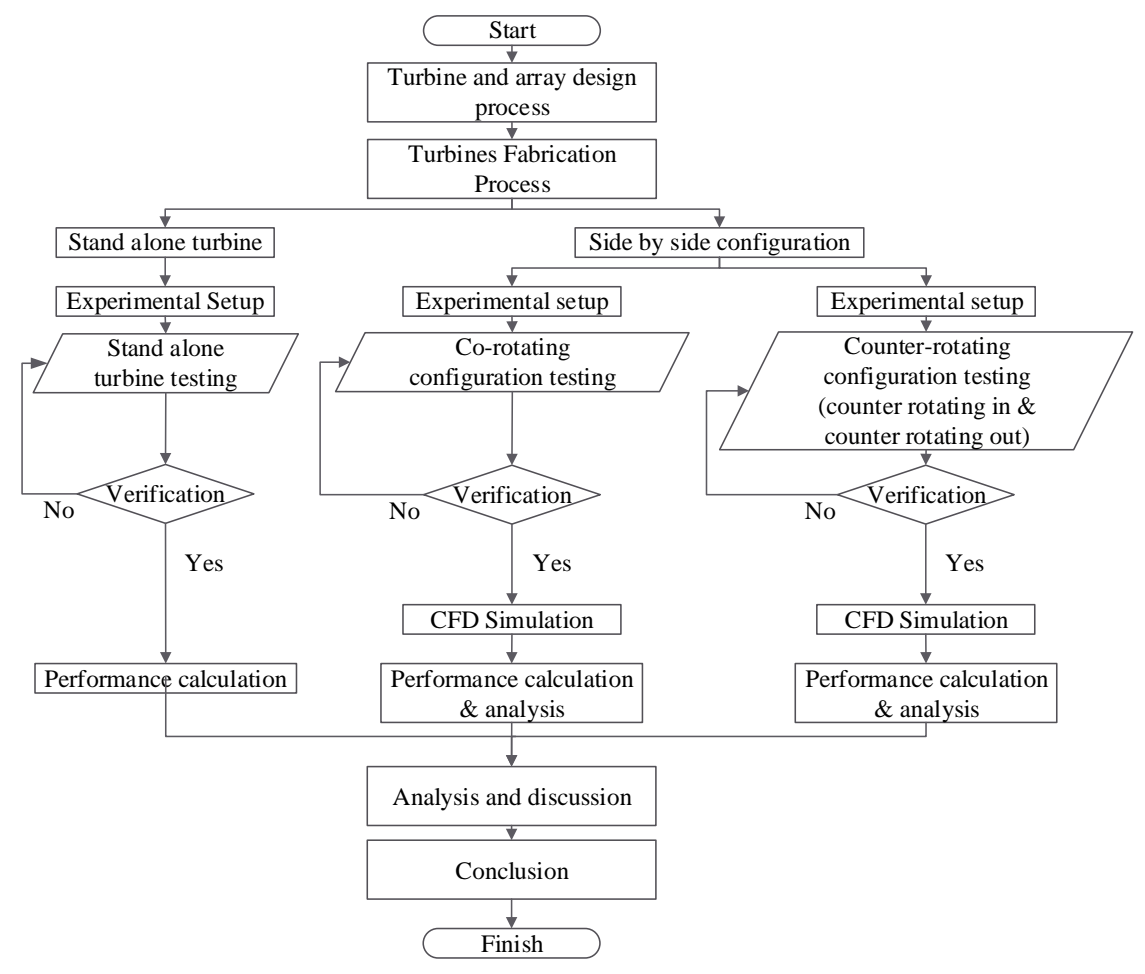

Figure 3. Research Methodology 
Turbine testing process consisted of three stages, i.e. stand-alone turbine testing, "co-rotating configuration" testing and "counter-rotating configuration" testing (consisting of "counter-rotating in" and "counter-rotating-out"), as illustrated in Figure 3. This turbine testing process was conducted to determine the performance of each configuration. The performance of each configuration was compared with the "stand-alone turbine" performance, hence the effect of side-by-side configuration on turbine's performance could be analyzed. Hereafter, the numerical simulation using Computational Fluid Dynamic method was carried out based on the experimental results.

\subsection{Turbines Array Testing Process}

Turbines array testing process was carried out to test the performance of each configuration. The testing process was conducted in Towing Tank Hydrodynamic Laboratory, Faculty of Marine Technology, Institut Teknologi Sepuluh Nopember, Surabaya. This Towing tank has $50 \mathrm{~m}$ length, 3 $\mathrm{m}$ width and $2 \mathrm{~m}$ height. Turbines were attached at cart which is located above the tank by using clamp F, as shown in Figure 4. Each turbine installation required four clamp F. Since the turbines were on overhanging position, the turbines should be firmly attached to prevent vibration which could damage turbines structure. Each turbine withdrawn with free stream velocity variations of $0.7 ; 1$ and $1.3 \mathrm{~m} / \mathrm{s}$.

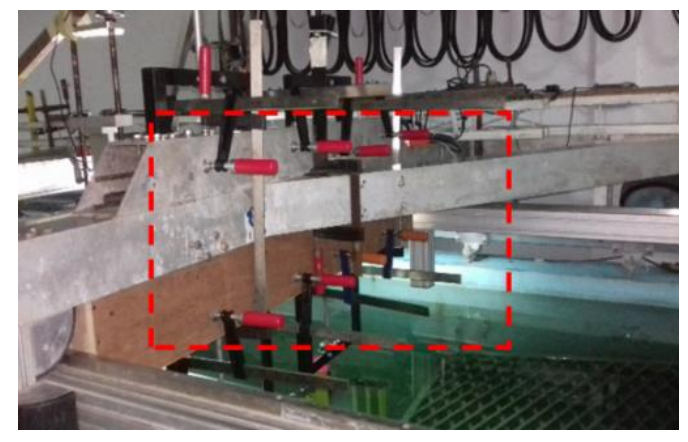

Figure 4. Turbine installation on Towing Tank's cart

The data obtained from the testing proses was rpm, which was recorded by camera. A tachometer was used in one turbine. Moreover, rpm data from tachometer measurement was used to validate the rpm gained form camera.

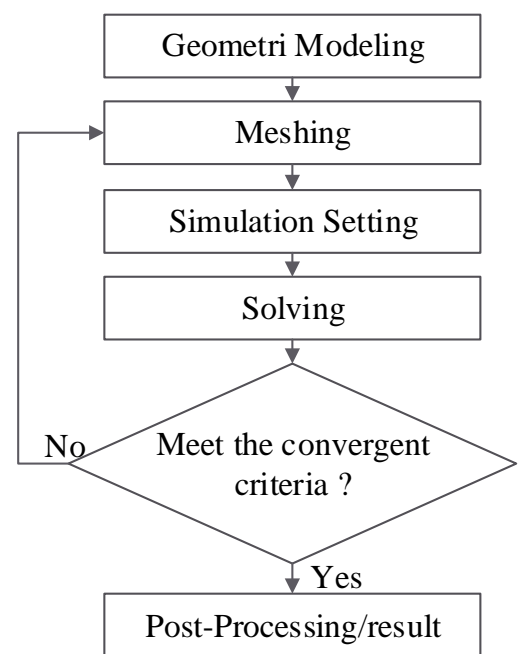

Figure 5. Simulation Processes 


\subsection{Numerical Simulation Set Up}

Since the limitations of experimental data, numerical simulation was performed to gain deep information on hydrodynamic interaction and other supplementary data. Numerical simulation was carried out using Computational Fluid Dynamic Method (CFD Method). CFD simulation method consists of several steps, i.e. pre-processing (Geometry modelling, meshing and simulation setting), processing (solving) and post-processing (result), as shown in Figure5.

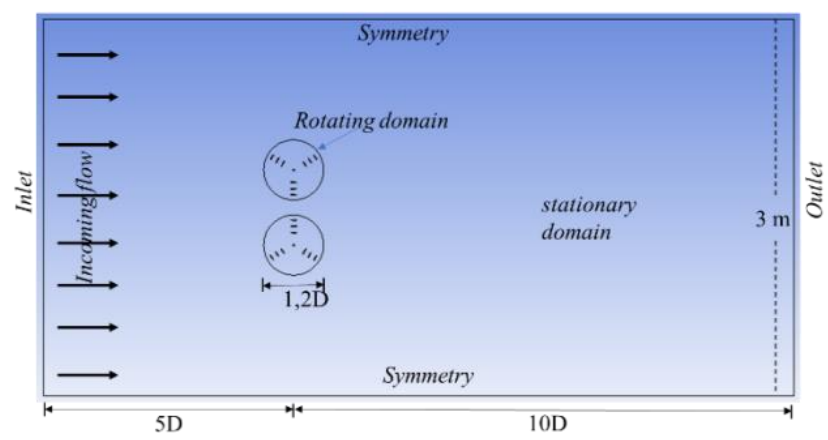

Figure 6. Computational domain

The CFD simulation employed in this study based on Raynold Average Navier-Stokes (RANS). RANS solver is a couple of flow solver where the momentum and continuity equation are solved simultaneously. This approach has less number of iteration and doesn't required pressure correction to maintain mass conservation, leading to more robust and accurate solver [15], [16].

The computational domain should be represented the real condition of testing field. In this numerical simulation, there were two types of computational domain, i.e. stationary domain (canal) and rotating domain (turbines). The stationary domain was 15D x 3 meter, with upstream distance of 5D and downstream distance of 10D [10], [17], as illustrated in Figure 6. D is turbine's diameter. Sliding mesh technique was employed in this numerical simulation. The inflation technique was used to refine the mesh quality.

Table 1. Boundary condition used

\begin{tabular}{|l|l|}
\hline \multicolumn{1}{|c|}{ Parameter } & \multicolumn{1}{c|}{ Condition } \\
\hline Turbulence model & k-omega SST \\
\hline Rotating domain & $\begin{array}{l}\text { Mesh motion with angular velocity adjusted to the experimental } \\
\text { results }\end{array}$ \\
\hline Boundary Condition & \\
\hline Inlet & $\begin{array}{l}\text { Velocity inlet } \\
\text { Normal to Boundary }\end{array}$ \\
\hline Outlet & Pressure outlet \\
\hline Blade/turbine & $\begin{array}{l}\text { Wall } \\
\text { Moving wall (Rotational) }\end{array}$ \\
\hline Symmetry & Symmetry \\
\hline Initialization method & Hybrid initialization \\
\hline
\end{tabular}

The selection of turbulence model should be considered before running the simulation. Many turbulence models have been developed aiming to get the best accuracy and the lowest computational requirement. $\mathrm{k}-\omega \mathrm{SST}$ is a promising model for turbine simulations. It is derived from eddy viscosity equation. It gives accurate prediction of the flow separation under adverse pressure gradients and has been successfully used in the CFD simulation of the wind or water turbines. In contrast, some 
applications have shown that the $\mathrm{k}-\varepsilon$ model has limitations in boundary layer flow with adverse pressure gradients [18]-[22]. Considering the advantages of k- $\omega$ SST, this study was utilized it. The boundary condition is described in Table 1 .

\section{Result and Discussion}

Hydrokinetic turbine is a promising energy harvesting system which converts hydro energy into electricity. The most developing hydrokinetic turbine technology, Darrieus Straight Blade is recommended to be applied in Indonesia, due to its reliability in extracting energy from low current velocity resource. The Darrieus development aims to enhance the power coefficient $(\mathrm{Cp})$ and selfstarting capability. The development efforts which is undertaken by previous research was the utilization of passive-pitch and cascaded blade mechanism. Both mechanisms increase torque and self-starting capability.

To meet the energy production demand, the hydrokinetic turbines are installed in an arrangement, called turbines array. The considered issues in turbine arrays design are turbines position in configuration, distance between turbines, turbines' rotational direction and so on. All mentioned highly affect the turbine performance, which directly corresponds to the array's performance. This research focuses on the effect of turbine's rotational direction on the turbine's and array's performance. To achieve the objectives, the turbine array testing was carried out in Towing Tank. To get deeper information, the numerical simulation was conducted using Computational Fluid Dynamic Method, aiming to analyse the hydrodynamic interaction around turbines.

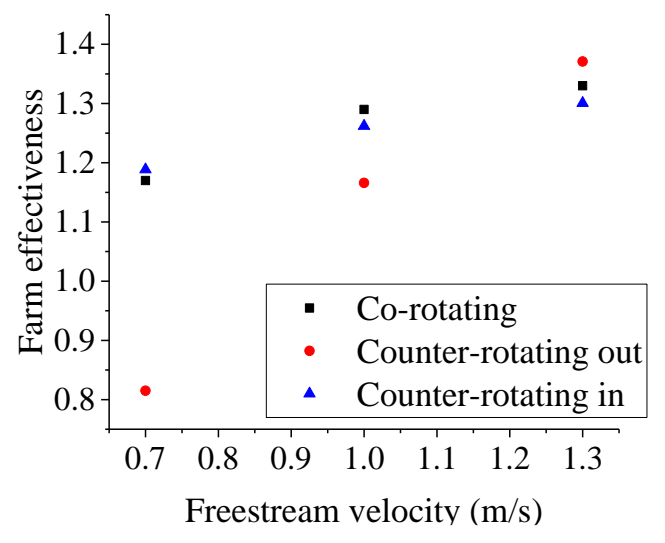

Figure 7. Farm effectiveness for each configuration

Freestream velocity affects the performance of each turbine. As the freestream velocity increase, the energy produced by the turbine increases, leading to the improvement of array performance. Increase in freestream velocity indicates the greater the hydrokinetic energy potential. Hence the chance of turbines to extract more energy is greater. Furthermore, the array performance is affected by the distance between turbines (cross-stream distance). Close distance between turbines could increase the intensity of hydrodynamic interaction, which tends to lead array performance incensement.

Due to the different hydrodynamic interaction, each configuration has different performance. Figure 7 exhibits array performance of each configuration. Array performance is represented by the value of farm effectiveness, which is mathematically expressed in equation (1). Farm effectiveness was calculated by comparing the power generated by the side-by-side turbines configuration with the power generated by two stand-alone turbines. The power which is produced by the stand-alone turbine represents the power generated by turbine if there is no hydrodynamic interaction. Meanwhile, the 
power generated by the side-by-side configuration ("co-rotating", "counter-rotating in" and "counter-rotating out") is affected by hydrodynamic interactions between turbines.

"Co-rotating" and "counter rotating-in" configuration have almost the same farm effectiveness, meanwhile the "counter-rotating out" configuration have the worst performance. This phenomenon is occurred because of the different flow superposition (between incoming flow and induced flow). The flow superposition for each configuration is distinguished clearly in Figure 8, which describes the hydrodynamic interaction at the freestream velocity of $1.3 \mathrm{~m} / \mathrm{s}$ and cross-stream distance of $1.5 \mathrm{D}$. For "counter-rotating out" configuration, the induced flow is in the opposite direction to the incoming flow, leading to the low flow velocity in the interaction zone (a zone that lies between turbine A and turbine B) than for the others. The flow velocity in the interaction zone for "co-rotating", "counterrotating out" and "counter-rotating in" is around $1.4-1.6 ; 1.2-1.6$ and $1.6-1.8 \mathrm{~m} / \mathrm{s}$, respectively. Since the induction flow and the incoming flow in the counter rotating-out are in the opposite direction, the flow superposition provides low velocity.

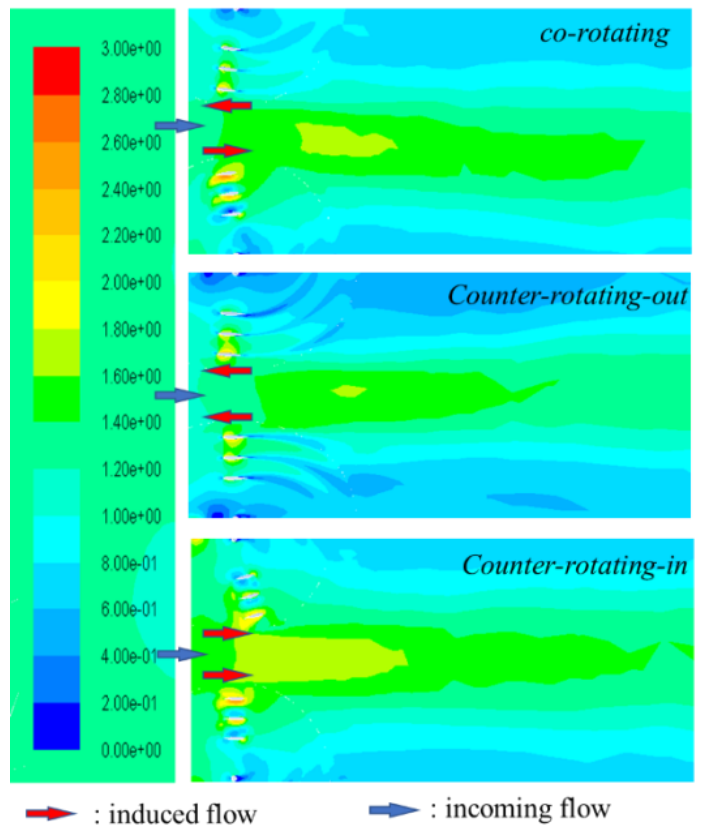

Figure 8 . Velocity counter in interaction zone for each configuration

Superposition of induction flow and freestream velocity lead to the flow acceleration in interaction zone. This acceleration depends on the rotational direction of each turbine. This phenomenon is called canal effect, or some literature calls it as jet-flow effect. The flow acceleration provides constructive effect on turbine performance, which is marked by $\mathrm{Cp}$ improvement. Hence, $\mathrm{Cp}$ of each turbine is higher than that for stand-alone turbine.

The turbulence intensity around the turbine is affected by the turbine's rotational direction. The turbulence intensity is the ratio of velocity fluctuation to the mean velocity at certain point. The flow quality subjected to the turbine is influenced by its turbulence characteristic, which takes effect on the array effectiveness. The turbulence intensity of "counter-rotating in" could be distinguished easily with other configuration, as it has the lowest turbulence intensity. Because the induction flow and the freestream flow are in the same direction, the velocity superposition yields in low flow fluctuation (low turbulence intensity). It is the lowest turbulence intensity among other configurations. This phenomenon indicates that flow in the interaction zone of "counter-rotating in" configuration tends to more stable, leading to the higher farm effectiveness. Due to the difference direction of incoming 
flow and induced flow, the turbulence intensity of "counter-rotating-out" configuration is relatively high and unstable.

The significant difference of turbulence intensity does not occur in the interaction zone, but in within the turbine. At the centre of the turbine $(\mathrm{X} / \mathrm{D}=0)$, the turbulence intensity of the "counterrotating out" configuration is greater than others. The turbulence intensity of the "counter-rotating out" even reaches $25 \%$. Meanwhile, the turbulence intensity of "co-rotating" is only $20 \%$ and $12.5 \%$. This distinction occurs due to difference induced flow of each configuration. The greatest turbulence intensity appears around the blades, especially in the trailing edge. Due to the flow separation in the trailing edge, the vortex structure is formed. Vortex is an unstable flow and characterize by high turbulence intensity.

The flow around vertical axis turbine is complex. The downstream blade and the upstream blade have difference characteristic. Due to energy extraction process in upstream, the downstream part obtains disturbed flow which consists of many vortex structures. The energy extraction process in downstream part carries more complex structure to the wake regions, causing high turbulence intensity. Furthermore, there is significant difference in turbulence intensity between "major lift production zone" and "minor lift production zone". The "major lift production zone" is zone where the most lift is produced. Thus, this area has big influence on turbine performance. "Major lift production zone" tends to gain higher turbulence intensity. "Major lift production zone" also considered by high flow velocity.

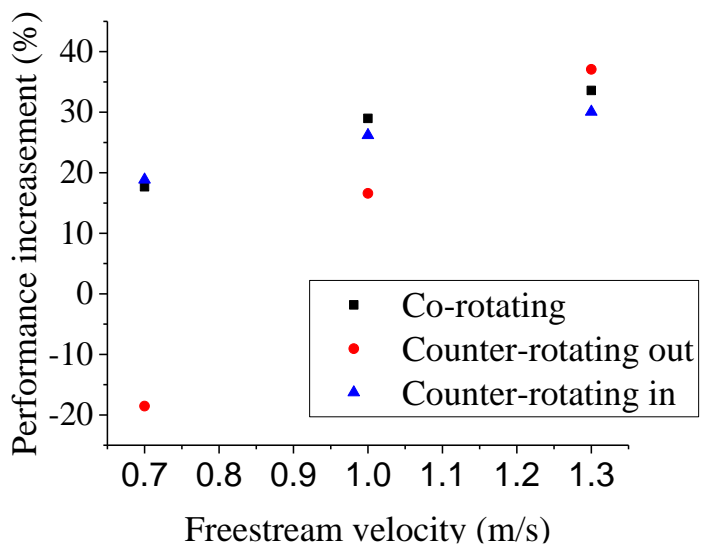

Figure 9. Performance increment

Turbulence formed around the turbine is categorized as weak turbulence, in which it easy to dissipate. This type of turbulence requires continues energy supply to maintain turbulence energy [23]. In this case, the energy is supplied by the vortex generated during turbine rotation. The vortex tends to move to the downstream, so that the turbulence in the downstream is more persistent (difficult to dissipate) than in the turbine side. As the result, the turbulence intensity in the downstream is higher than in the inlet and interaction zone. The numerical simulation confirmed that the turbulence in the wake region (downstream) is be dissipated in certain downstream distance. For "co-rotating" and "counter-rotating out" configuration, the turbulence is dissipated at 5.5 D. Meanwhile, the turbulence in "counter-rotating in" is dissipated at 5D, where D is turbines' diameter. This notifies that the downstream turbine should be installed beyond 5D to minimize the adverse effects of turbulence, so that downstream turbines could work optimally. Turbulence conditions in the downstream area is very important consideration for designing turbine configuration which consists of multiple rows.

The installation of turbines in side-by-side configuration provides improvement in turbine performance. This improvement occurs for all designed configurations. Due to increment of flow 
interaction intensity as the increment of freestream velocity, the performance of each turbine also increases with the increase of freestream velocity. At freestream velocity of $1.3 \mathrm{~m} / \mathrm{s}$, the performance increasement reaches $33.6 \%$ for "co-rotating configuration", 37\% for "counter-rotating out" configuration and $30.1 \%$ for "counter-rotating in" configuration. However, at low freestream velocity $(0.7 \mathrm{~m} / \mathrm{s})$, the "counter-rotating out" configuration provides poor performance, marked by a performance increasement of $-18 \%$.

Hydrokinetic turbine is a modern technology which could be installed in various installation site, e.g. canal, river, ocean or estuary. For ocean application where the flow direction is unpredictable, the "co-rotating" configuration is recommended since it provides the same effect even though the direction of incoming flow is change. But the installation of "counter-rotating in" is risky because it will systematically produce difference hydrodynamic effect when the incoming flow change, leading to the decrement of farm effectiveness.

\section{Conclusion}

Array testing and numerical simulation confirmed that the installation turbines in array configuration could improve the performance of each turbine, leading to the enhancement of farm effectiveness. This performance improvement occurs as the effect of hydrodynamic interaction between turbines. The hydrodynamic interaction causes flow superposition between induced flow and incoming flow. Different configurations have different hydrodynamic interaction, resulting different effect on farm improvement. Furthermore, the close cross-stream distance causes flow acceleration in the interaction zone, which is known as canal effect or jet-type flow field. The "corotating" and "counter-rotating in" configuration has better performance than "counter-rotating out". The farm effectiveness of "co-rotating" configuration and "counter-rotating in" is 1.33 and 1.37, respectively (at freestream velocity of $1.3 \mathrm{~m} / \mathrm{s}$ and cross-stream distance of 1.5D). Installation of sideby-side configuration provides a performance improvement of more than $30 \%$ at a freestream speed of $1.3 \mathrm{~m} / \mathrm{s}$. However, "co-rotating" configuration is recommended for array installation in unstable flow direction resource, where the incoming flow direction could not be predicted.

\section{References}

[1] M. J. Khan, M. T. Iqbal, and J. E. Quaicoe, 'River current energy conversion systems: Progress, prospects and challenges', Renew. Sustain. Energy Rev., vol. 12, no. 8, pp. 2177-2193, 2008.

[2] Y. Li, 'On the definition of the power coefficient of tidal current turbines and efficiency of tidal current turbine farms', Renew. Energy, vol. 68, pp. 868-875, 2014.

[3] J. Bartl, Wake Measurements Behind an Array of Tow Model Wind Turbines, no. October. 2011.

[4] B. Sanderse, 'Aerodynamics of wind turbine wakes: Literature review', Energy Res. Cent. Netherlands, pp. 1-46, 2009.

[5] V. Movahed, H. Sharafi, M. H. Baziar, and H. Shahnazari, 'An Experimental Investigation on the Effects of Turbine Rotation Directions on the Wake Interference of Wind Turbines', 5th Int. Conf. Earthq. Eng., no. January, pp. 1-16, 2011.

[6] R. Conzemius, 'Wind turbine and sodar observations of wakes in a large wind farm', in 19th Symposium on Boundary Layers and Turbulence, 2010, p. 7.

[7] W. Yuan, O. Ahmed, W. Tian, and H. Hu, 'An Experimental Investigation on the Effect of Turbine Rotation Direction on the Wake Interference of Wind Turbine', in Aiaa, 2013, vol. 3815, no. January, pp. 1-18.

[8] N. J. Lee, I. C. Kim, C. G. Kim, B. S. Hyun, and Y. H. Lee, 'Performance study on a counter-rotating tidal current turbine by CFD and model experimentation', Renew. Energy, vol. 79, no. 1, pp. 122-126, 2015.

[9] G. Tescione, C. J. Simão Ferreira, and G. J. W. van Bussel, 'Analysis of a free vortex wake model for the study of the rotor and near wake flow of a vertical axis wind turbine', Renew. Energy, vol. 87, pp. 552-563, 2016.

[10]A. Posa, C. M. Parker, M. C. Leftwich, and E. Balaras, 'Wake structure of a single vertical axis wind turbine', Int. J. Heat Fluid Flow, vol. 61, pp. 75-84, 2016.

[11]I. Ross and A. Altman, 'Wind tunnel blockage corrections: Review and application to Savonius vertical-axis wind turbines', J. Wind Eng. Ind. Aerodyn., vol. 99, no. 5, pp. 523-538, 2011. 
[12]H. Beri and Y. Yao, 'Double Multiple Streamtube Model and Numerical Analysis of Vertical Axis Wind Turbine', Energy Power Eng., vol. 3, no. 3, pp. 262-270, 2011.

[13]I. Paraschivoiu, F. Delclaux, P. Fraunié, and C. Béguier, 'Aerodynamic Analysis of the Darrieus Wind Turbines Including Secondary Effects', J. Energy, vol. 7, no. 5, pp. 416-422, 1983.

[14]S. Eriksson, H. Bernhoff, and M. Leijon, 'Evaluation of different turbine concepts for wind power', Renew. Sustain. Energy Rev., vol. 12, no. 5, pp. 1419-1434, 2008.

[15]S. Derakhshan, M. Ashoori, and A. Salemi, 'Experimental and numerical study of a vertical axis tidal turbine performance', Ocean Eng., vol. 137, no. March, pp. 59-67, 2017.

[16]S. Derakhshan and N. Kasaeian, 'Optimization, Numerical, and Experimental Study of a Propeller Pump as Turbine', J. Energy Resour. Technol., vol. 136, no. 1, p. 12005, 2014.

[17]G. Zhao, R. S. Yang, Y. Liu, and P. F. Zhao, 'Hydrodynamic performance of a vertical-axis tidal-current turbine with different preset angles of attack', J. Hydrodyn., vol. 25, no. 2, pp. 280-287, 2013.

[18]R. Hantoro, I. K.A.P Utama, E. Erwandi, and A. Sulisetyono, 'An Experimental Investigation of Passive VariablePitch Vertical-Axis Ocean Current Turbine', ITB J. Eng. Sci., vol. 43, no. 1, pp. 27-40, 2011.

[19]F. R. Menter, 'Two-equation eddy-viscosity turbulence models for engineering applications', AIAA J., vol. 32, no. 8, pp. 1598-1605, 1994.

[20]P. Marsh, D. Ranmuthugala, I. Penesis, and G. Thomas, 'Numerical investigation of the influence of blade helicity on the performance characteristics of vertical axis tidal turbines', Renew. Energy, vol. 81, pp. 926-935, 2015.

[21]B. K. Kirke and L. Lazauskas, 'Limitations of fi xed pitch Darrieus hydrokinetic turbines and the challenge of variable pitch', Renew. Energy, vol. 36, no. 3, pp. 893-897, 2011.

[22]B. Yang and C. Lawn, 'Fluid dynamic performance of a vertical axis turbine for tidal currents q', Renew. Energy, vol. 36, no. 12, pp. 3355-3366, 2011.

[23] H. Tennekes and J. . Lumley, A first course in turbulence. Cambridge, Massachusetts, London: MIT Press. 